\title{
Mitral valve repair and surgical ablation for atrial functional mitral regurgitation
}

\author{
Jinmiao Chen ${ }^{1 \#}$, Yulin Wang ${ }^{1 \#}$, Minzhi Lv ${ }^{2 \#}$, Zhaohua Yang ${ }^{1}$, Shijie Zhu ${ }^{1}$, Lai Wei ${ }^{1}$, Tao Hong ${ }^{1}$, \\ Wenjun Ding ${ }^{1}$, Yi Lin ${ }^{1}$, Chunsheng Wang ${ }^{1}$ \\ ${ }^{1}$ Department of Cardiac Surgery, Zhongshan Hospital, Fudan University, Shanghai, China; ${ }^{2}$ Department of Biostatistics, Zhongshan Hospital, Fudan \\ University, Shanghai, China \\ Contributions: (I) Conception and design: J Chen, Y Lin, C Wang; (II) Administrative support: L Wei, W Ding, T Hong, C Wang; (III) Provision of \\ study materials or patients: L Wei, W Ding, T Hong, C Wang; (IV) Collection and assembly of data: J Chen, Y Wang, M Lv, Z Yang, S Zhu; (V) \\ Data analysis and interpretation: J Chen, Y Wang, M Lv; (VI) Manuscript writing: All authors (VII) Final approval of manuscript: All authors. \\ "These authors contributed equally to this work. \\ Correspondence to: Yi Lin, MD, PhD; Chunsheng Wang, MD. Department of Cardiac Surgery, Zhongshan Hospital, Fudan University, 180 Fenglin \\ Road, Shanghai 200032, China. Email: lin.yi@zs-hospital.sh.cn; wang.chunsheng@zs-hospital.sh.cn.
}

Background: This observational study aimed to share our experience in the surgical management of atrial functional mitral regurgitation (AFMR).

Methods: We retrospectively identified 82 AFMR patients (63.6 7.7 years) from June 2008 to November 2018 at our institution. Of these patients, $72.0 \%$ of them were classified as NYHA functional class III/IV, and all of them had persistent AF. All patients underwent mitral valve (MV) repair, and 52 (63.4\%) received concomitant surgical ablation (SA). Patients were followed up for $26.1 \pm 27.6$ months, and postoperative mitral regurgitation (MR) was assessed by echocardiography.

Results: There was no in-hospital mortality. The overall 1-year and 3-year survival rates were $97.5 \%$ and $92.9 \%$, respectively, and $96.1 \%$ of patients recovered to NYHA functional class I/II at the latest followup. The left atrium $(\mathrm{LA})$ diameter $(\mathrm{P}<0.001)$, left ventricular $(\mathrm{LV})$ end-diastolic diameter $(\mathrm{P}<0.001), \mathrm{LV}$ end-systolic diameter (LVESD) $(\mathrm{P}<0.001)$ and pulmonary artery pressure $(\mathrm{P}=0.006)$ significantly decreased postoperatively. The overall 1-year and 3-year freedom from recurrent $\mathrm{MR}$ rates were $94.3 \%$ and $65.3 \%$, respectively, and a significant difference was found between the SA group and the non-SA group (93.8\% and $93.8 \%$ vs. $95.5 \%$ and $44.2 \%, \mathrm{P}=0.035$ ). In a subgroup analysis, this significant difference was only found in the small LA group $(\leq 60 \mathrm{~mm})$.

Conclusions: Our results suggest that MV repair for AFMR is safe and effective. It improves heart failure symptoms and results in reverse-remodeling of both the LA and LV. Concomitant SA might benefit patients in terms of recurrent MR, especially in the small LA group ( $\leq 60 \mathrm{~mm})$.

Keywords: Atrial fibrillation (AF); mitral regurgitation (MR); mitral valve repair (MV repair); surgical ablation (SA)

Submitted Mar 29, 2020. Accepted for publication Aug 28, 2020.

doi: 10.21037/atm-20-2958

View this article at: http://dx.doi.org/10.21037/atm-20-2958

\section{Introduction}

Atrial functional mitral regurgitation (AFMR) refers to atrial fibrillation (AF)-induced functional mitral regurgitation (MR), with structurally normal mitral leaflets (1-4). Patients often present with relatively normal left ventricular (LV) size and function, but with an enlarged left atrium (LA) and mitral annulus. The potential underlying mechanisms of AFMR may be related to an AF-induced enlarged mitral annulus, atriogenic tethering of the posterior mitral leaflet, and insufficient leaflet remodeling (5-8). While this pathology is relatively rare and still poorly described, it is becoming increasingly recognized by cardiologists and 
cardiac surgeons in recent years.

The optimal treatment algorithm for AFMR remains unclear. Some studies have demonstrated that restoration of sinus rhythm by catheter ablation can mitigate MR $(1,9)$. However, high rate of residual MR at 1-year follow-up have been reported even after the restoration of sinus rhythm (1). Therefore, for AF patients with significant functional MR and progressive heart failure symptoms, catheter ablation may not be sufficient, due to irreversible enlargement of the mitral annulus $(10,11)$. Very few studies have reported on the surgical interventions for AFMR (12). This study aimed to report our clinical experience in the surgical management of AFMR. We present the following article in accordance with the STROBE reporting checklist (available at: http:// dx.doi.org/10.21037/atm-20-2958).

\section{Methods}

\section{Study population}

We retrospectively enrolled AFMR patients at our center from June 2008 to November 2018 with the following inclusion criteria: (I) persistent AF; (II) at least moderateto-severe central MR; (III) LV ejection fraction (LVEF) $>50 \%$; (IV) LV end-diastolic diameter (LVEDD) $<60 \mathrm{~mm}$; (V) LV end-systolic diameter (LVESD) $<45 \mathrm{~mm}$. All enrolled patients had AF first, then gradually developed moderate-to-severe MR. AF was defined as persistent if typical AF episodes lasted $\geq 7$ days (13). Patients with mitral leaflet prolapse, rheumatic mitral valve (MV) disease, infective endocarditis, coronary artery disease, congenital heart disease, dilated cardiomyopathy, hypertrophic cardiomyopathy, or other structural heart valve diseases were excluded. The study was conducted in accordance with the Declaration of Helsinki (as revised in 2013). The study protocol was approved by the Ethics Committee of Zhongshan Hospital, Fudan University. Because of the retrospective nature of the research, the requirement for informed consent was waived.

\section{Surgical technique}

All patients underwent a median sternotomy and moderate hypothermic cardiopulmonary bypass. Cardiopulmonary bypass was established via aorto-bicaval cannulation. For surgical ablation (SA), the standard Cox-Maze IV procedure was performed using a Medtronic radiofrequency ablation kit (14-16). The LA appendage was routinely cut and oversewn. The MV was exposed through the inter-atrial groove or atrial septal approach if the tricuspid valve needed intervention. An appropriately sized posterior annuloplasty ring (Cosgrove-Edwards, Edwards Lifesciences) or complete annuloplasty ring (Physio or Physio II, Edwards Lifesciences) was implanted. Subsequently, valve competency was tested by injecting cold saline into the LV across the MV. Concomitant tricuspid annuloplasty was performed if the preoperative tricuspid annulus was larger than $40 \mathrm{~mm}$. Intraoperative transesophageal echocardiography was performed in all cases to evaluate valve competency after repair.

\section{Medication management}

Anti-arrhythmic drugs were administered to all patients after surgery for at least 3 months unless contraindicated. Amiodarone was the first choice. If patients exhibited sinus rhythm at 3 months after surgery, the use of anti-arrhythmic drugs was discontinued. Warfarin was given to all patients with a target international normalized ratio (INR) of 1.8-2.5 in the first 3 months but was stopped if they reverted to sinus rhythm.

\section{Follow-up}

Follow-up included outpatient visits and telephone calls. Overall survival (OS) was defined as the interval between the date of surgery and the date of any-cause death. Patients received transthoracic echocardiography (TTE) during follow-up. The latest follow-up TTEs were used as for the postoperative echocardiographic data.

\section{Statistical analysis}

Continuous normally distributed variables were presented as the mean \pm standard deviation, whereas non-normally distributed variables were presented as median and first and third quartiles. The assumption of normality was assessed by the Shapiro-Wilk test. Continuous normally distributed variables were compared by using Student's $t$-test. Nonnormally distributed variables were compared by using the nonparametric Wilcoxon rank-sum test. Paired continuous data were compared by using the paired $t$-test or Wilcoxon signed-rank test. Categorical variables were presented as a proportion and compared between groups by using the chisquared test or Fisher's exact test where appropriate.

The primary outcome was recurrent MR (greater than 
a mild-to-moderate degree). Survival distributions and freedom from recurrent MR were calculated according to the Kaplan-Meier method and compared by using the Log-Rank test. Cox proportional hazards models were constructed to calculate the hazard ratios (HRs). Also, we used the multivariable model adjusted by propensity score (PS) and the inverse probability of treatment weighting (IPTW) Cox regression model to reduce bias and estimate the exact effect of SA treatment. IPTW was calculated based on PS to create a pseudo-population in which the distribution of measured baseline covariates was independent of treatment (17). The PS model of SA was constructed by using the multivariable logistic regression model, which included age, gender, preoperative NYHA functional class, AF duration, annuloplasty ring type, annuloplasty ring size, previous ablation, and preoperative MR degree.

For all analyses, tests were two-tailed, and $\mathrm{P}$ values $<0.05$ were considered statistically significant. All data were analyzed using the JMP System software (version 14.0, SAS Institute Inc., Cary, NC, USA) and R software (version 3.5.3, R Foundation for Statistical Computing, Vienna, Austria).

\section{Results}

\section{Patient characteristics}

Patient characteristics are summarized in Table 1 . The mean age of patients was $63.6 \pm 7.7$ years, which suggested that AFMR patients were typically elderly. The predominant symptom was dyspnea (82.9\%) and palpitation (62.2\%). At admission, $72.0 \%$ of patients presented with NYHA function class III/IV. All patients had persistent AF, and the diagnosis time was $24(5.8-54.8)$ months. Three patients $(3.7 \%)$ had previous catheter ablation therapy. By comparing the preoperative echocardiographic data between the SA group and the non-SA group, we found that the non-SA group tended to larger LAs $(51.7 \pm 8.3 \mathrm{vs}$. $58.1 \pm 9.0 \mathrm{~mm}, \mathrm{P}=0.003$ ) (Table 1). Also, a more significant proportion of severe MR was found in the non-SA group.

\section{Surgical treatment and outcomes}

Table 2 shows the surgical data of this cohort. All 82 patients underwent MV repair, and $63.4 \%$ received concomitant SA. Consequently, $65.4 \%$ of patients had restored sinus rhythm in the SA group, compared to only $10 \%$ of patients in the non-SA group $(\mathrm{P}<0.001)$.
Posterior annuloplasty rings were implanted in 47 (57.3\%) patients with a mean size of $30.1 \pm 2.0 \mathrm{~mm}$, while complete annuloplasty rings were used in the rest of the patients with a mean size of $29.2 \pm 1.8 \mathrm{~mm}(\mathrm{P}=0.027)$. Additionally, $87.8 \%$ of patients underwent concomitant tricuspid annuloplasty. The mean cardiopulmonary bypass time and cross-clamp time in all patients were $110.4 \pm 32.8$ and $61.2 \pm 22.5$ minutes, respectively. The concomitant SA increased cardiopulmonary bypass time and crossclamp time (Table 2). Intraoperative transesophageal echocardiography showed that MV repair was satisfactory without significant systolic anterior motion. There were no cases of in-hospital death, cerebrovascular events, deep sternal wound infection, or acute renal impairment necessitating hemofiltration after surgery.

The mean follow-up time after surgery was $26.1 \pm 27.6$ months. There were 4 all-cause deaths after discharge: 1 patient died at 30 months due to reoperation for severe recurrent MR, 1 patient died at 55 months because of myocardium infarction and cerebral infarction, and 2 patients died at 1 month for reasons unknown. The overall 1-year and 3-year survival rates were $97.5 \%$ (95\% CI: $94.1-100.0 \%)$ and $92.9 \%$ (95\% CI: $83.9-100.0 \%)$, respectively, and no significant difference was found between the SA group (3/52) and the non-SA group (1/30) (Log-rank test: $\mathrm{P}=0.439)$. Also, $96.1 \%$ of the surviving patients recovered to NYHA functional class I/II at the latest follow-up $(\mathrm{P}<0.001$ vs. preoperatively).

\section{Cardiac reverse-remodeling}

All patients had at least one TTE examination during follow-up, and the mean TTE follow-up time was 18.6 \pm 24.7 months. The follow-up TTE data are summarized in Table 3. Compared to preoperative echocardiography, the LA diameter (LAD) $(\mathrm{P}<0.001)$, LVEDD $(\mathrm{P}<0.001)$, LVESD $(\mathrm{P}<0.001)$ and pulmonary artery pressure $(\mathrm{P}=0.006)$ significantly decreased postoperatively, which indicated reverse-remodeling of both the LA and LV. This significant reverse-remodeling was found both in the SA group and the non-SA group (Table 3).

\section{Recurrent MR}

Ten patients were found to have recurrent MR (greater than a mild-to-moderate degree) during follow-up. The overall 1-year and 3-year freedom from recurrent MR rates were 94.3\% (95\% CI: $88.2-100.0 \%$ ) and $65.3 \%$ (95\% CI: $47.8-$ 
Table 1 Patients baseline characteristics

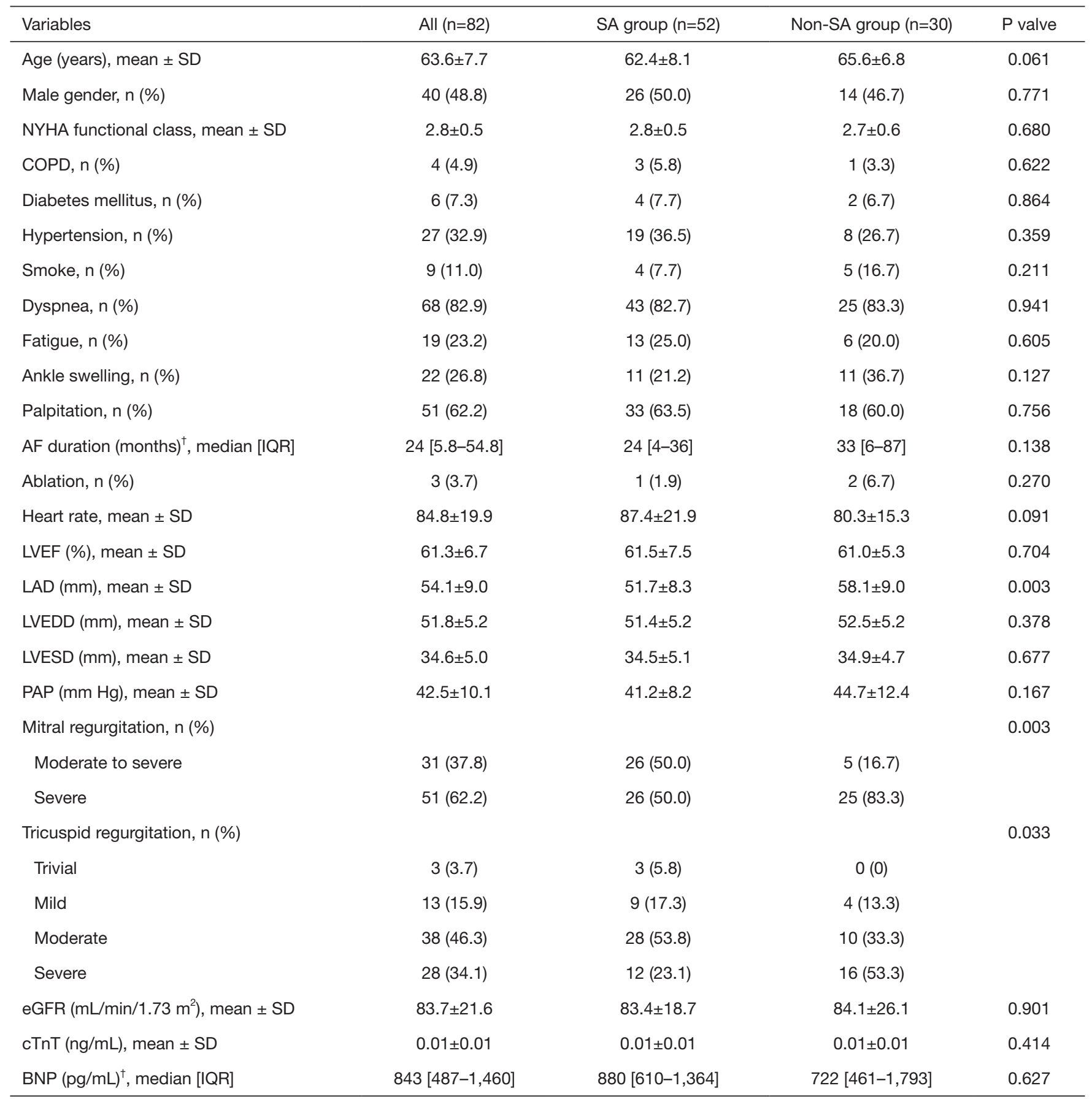

${ }^{\dagger}$, compared by use of the nonparametric Wilcoxon rank-sum test. BNP, type B natriuretic peptide; COPD, chronic obstructive pulmonary disease; eGFR, estimated glomerular filtration; NYHA, New York Heart Association; IQR, interquartile range; SA, surgical ablation; SD, standard deviation; LVEF, left ventricular ejection fraction; LAD, left atrial diameter; LVEDD, left ventricular end-diastolic diameter; LVESD, left ventricular end-systolic diameter; PAP, pulmonary artery pressure. 
Table 2 Operative details and postoperative outcomes

\begin{tabular}{|c|c|c|c|c|}
\hline Variables & All $(n=82)$ & SA group $(n=52)$ & Non-SA group $(n=30)$ & $P$ valve \\
\hline Cross-clamp time (min), mean $\pm \mathrm{SD}$ & $61.2 \pm 22.5$ & $68.5 \pm 18.7$ & $48.0 \pm 22.7$ & $<0.001$ \\
\hline Mitral annuloplasty ring, $\mathrm{n}(\%)$ & & & & 0.194 \\
\hline Posterior ring & $47(57.3)$ & $27(51.9)$ & $20(66.7)$ & \\
\hline Mitral ring size $(\mathrm{mm})$, mean $\pm \mathrm{SD}$ & $29.7 \pm 2.0$ & $29.3 \pm 1.8$ & $30.5 \pm 2.0$ & 0.007 \\
\hline Tricuspid valve repair, n (\%) & $72(87.8)$ & $43(82.7)$ & $29(96.7)$ & 0.063 \\
\hline Operative mortality, n (\%) & $0(0)$ & $0(0)$ & $0(0)$ & 1.000 \\
\hline Ventilation time $(\text { hours })^{\dagger}$, median [IQR] & $12[12-24]$ & $12[12-24]$ & 12 [12-24] & 0.425 \\
\hline Postoperative hospital stay $(\mathrm{d})^{\dagger}$, median (IQR) & $8.0(7.0-9.0)$ & $8.0(6.0-9.0)$ & $8.0(7.0-9.0)$ & 0.632 \\
\hline
\end{tabular}

${ }^{\dagger}$, compared by use of the nonparametric Wilcoxon rank-sum test. CPB, cardiopulmonary bypass; ICU, intensive care unit; IQR, interquartile range; SA, surgical ablation; SD, standard deviation

Table 3 Follow-up echocardiographic outcomes

\begin{tabular}{|c|c|c|c|c|c|c|c|c|c|c|c|}
\hline Variables & All $(n=73)$ & $\begin{array}{l}\text { SA group I } \\
\quad(n=45)\end{array}$ & $\begin{array}{l}\text { Non-SA group } \\
\qquad(n=28)\end{array}$ & $\begin{array}{l}\text { Difference } \\
(95 \% \mathrm{Cl})\end{array}$ & $P$ valve & $\begin{array}{l}\text { Difference } \\
(95 \% \mathrm{Cl})^{\$}\end{array}$ & $\begin{array}{c}P \\
\text { valve }^{\$}\end{array}$ & $\begin{array}{l}\text { Difference } \\
(95 \% \mathrm{Cl})^{\#}\end{array}$ & P valve ${ }^{\#}$ & $\begin{array}{l}\text { Difference } \\
(95 \% \mathrm{Cl})^{\&}\end{array}$ & $P$ valve ${ }^{\&}$ \\
\hline LVEF (\%) & $61.8 \pm 8.0$ & $62.2 \pm 8.0$ & $61.2 \pm 8.1$ & $\begin{array}{c}1.0 \\
(-2.8,4.9)\end{array}$ & 0.591 & $\begin{array}{c}-1.0 \\
(-2.8,0.9)\end{array}$ & 0.306 & $\begin{array}{c}-1.4 \\
(-3.8,1.0)\end{array}$ & 0.260 & $\begin{array}{c}-0.3 \\
(-3.4,2.7)\end{array}$ & 0.832 \\
\hline LAD (mm) & $49.7 \pm 9.5$ & $46.8 \pm 7.6$ & $54.4 \pm 10.4$ & $\begin{array}{c}-7.5 \\
(-12.1,-3.0)\end{array}$ & 0.002 & $\begin{array}{c}4.8 \\
(3.4,6.1)\end{array}$ & $<0.001$ & $\begin{array}{c}5.2 \\
(3.7,6.8)\end{array}$ & $<0.001$ & $\begin{array}{c}4.0 \\
(1.6,6.4)\end{array}$ & 0.002 \\
\hline LVESD (mm) & $32.8 \pm 5.5$ & $32.6 \pm 5.6$ & $33.0 \pm 5.4$ & $\begin{array}{c}-0.3 \\
(-3.0,2.3)\end{array}$ & 0.797 & $\begin{array}{c}2.2 \\
(1.1,3.3)\end{array}$ & $<0.001$ & $\begin{array}{c}2.4 \\
(0.9,3.8)\end{array}$ & 0.002 & $\begin{array}{c}2.0 \\
(0.2,3.8)\end{array}$ & 0.029 \\
\hline PAP $(\mathrm{mm} \mathrm{Hg})$ & $38.9 \pm 11.3$ & $37.9 \pm 8.6$ & $40.5 \pm 14.6$ & $\begin{array}{c}-2.7 \\
(-8.8,3.5)\end{array}$ & 0.386 & $\begin{array}{c}3.5 \\
(1.1,6.0)\end{array}$ & 0.006 & $\begin{array}{c}3.3 \\
(0.1,6.6)\end{array}$ & 0.046 & $\begin{array}{c}3.8 \\
(-0.2,7.7)\end{array}$ & 0.059 \\
\hline
\end{tabular}

The data were presented as the mean \pm standard deviation. ${ }^{\$}$, the comparison between preoperative and follow-up data in all patients. ${ }^{\#}$, the comparison between preoperative and follow-up data in SA group. " , the comparison between preoperative and follow-up data in non-SA group. $\mathrm{Cl}$, confidence interval; LVEF, left ventricular ejection fraction; LAD, left atrial diameter; LVEDD, left ventricular end-diastolic diameter; LVESD, left ventricular end-systolic diameter; PAP, pulmonary artery pressure; SA, surgical ablation.

$89.1 \%)$, respectively, and a significant difference was found between the SA group and the non-SA group $(93.8 \%$ and $93.8 \%$ vs. $95.5 \%$ and $44.2 \%, \mathrm{P}=0.035$ ) (Figure 1 ). Also, the recurrent $M R$ rate was found to be different between the small LA group ( $\leq 60 \mathrm{~mm}$ ) and large LA group $(>60 \mathrm{~mm})$, though this did not reach statistical significance (Log-rank test: $\mathrm{P}=0.064$ ) (Figure 2). In order to investigate the exact effect of SA on recurrent MR, we used a multivariable model adjusted by PS and IPTW Cox regression models to reduce bias. A significant interaction was found between SA and preoperative LAD in the crude model, PS-adjusted model, and PS IPTW model (Table 4). In practice, a higher rate of SA tended to be performed in the small LA group compared to the large LA group $(47 / 69,68.1 \%$ vs. 5/13, 

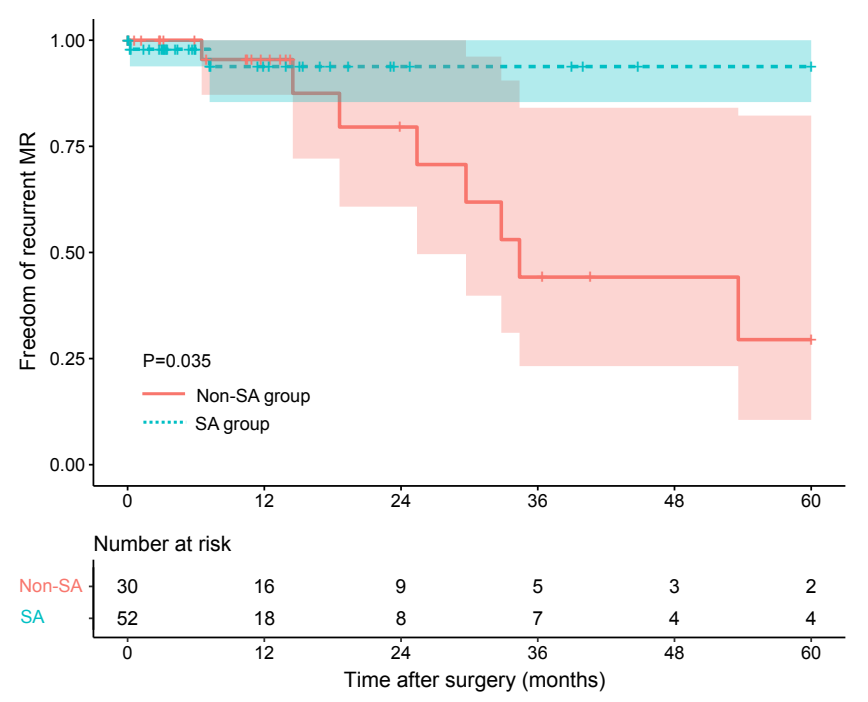

Figure 1 Kaplan-Meier curves depicting freedom from recurrent MR for the SA group and the non-SA group. Shadings indicate 95\% confidence intervals. MR, mitral regurgitation; SA, surgical ablation.
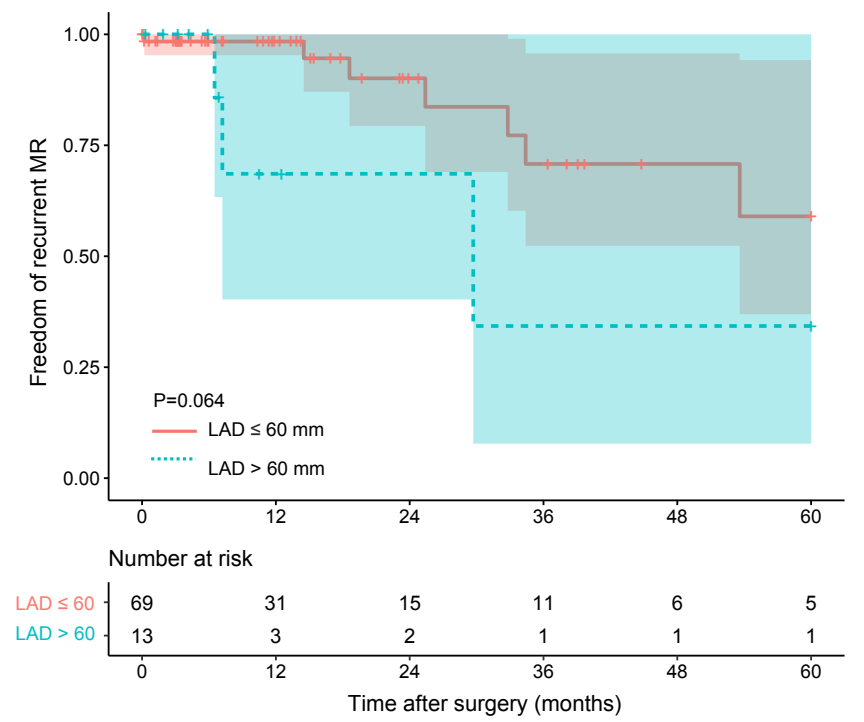

Figure 2 Kaplan-Meier curves depicting freedom from recurrent $M R$ for the $\mathrm{LAD} \leq 60 \mathrm{~mm}$ group and the LAD $>60 \mathrm{~mm}$ group. Shadings indicate $95 \%$ confidence intervals. LAD, left atrium diameter; MR, mitral regurgitation.

$38.5 \%, \mathrm{P}=0.042$ ). Subsequently, we performed a subgroup analysis and found that the significant effect of SA on recurrent MR was only in the small LA group and not in the large LA group (Table 5 and Figure 3).

\section{Discussion}

This study aimed to share our observational clinical experience in the surgical management of AFMR. The mid-term outcomes of MV repair for AFMR demonstrate its safety and efficacy by improving heart failure symptoms and inducing reverse-remodeling in both the LA and LV. Furthermore, we found that a concomitant SA procedure may reduce recurrent MR, especially in the small LA group ( $\leq 60 \mathrm{~mm})$.

$\mathrm{AF}$ is a common cardiac rhythm disorder, found predominantly in elderly patients and heart failure patients. In clinical practice, AF patients occasionally present with moderate-to-severe $\mathrm{MR}(2,6) . \mathrm{AF}$ is a known cause of $\mathrm{MR}$ and vice versa, although sometimes it is difficult to identify which pathology manifests first. AFMR patients often present with chronic heart failure symptoms, a progressive disease associated with poor prognosis $(10,11,18)$. Vohra and colleagues reported in their case series that $85 \%$ of patients had NYHA functional class III/IV symptoms (12). This is comparable to our cohort, where $82.9 \%$ of patients presented with dyspnea, and $72.0 \%$ of them were NYHA functional class III/IV.

The optimal treatment for AFMR is still unknown due to a poor understanding of the underlying pathological mechanisms. AF can beget LA enlargement and succeeds in mitral annular dilatation (19). In the context of AFMR, the mitral leaflets are usually structurally normal but morphologically enlarged (6). This means that there are enough leaflets for coaptation if annuloplasty rings are used to downsize the mitral annulus. Some studies have reported on MV repair for AFMR, but the sample sizes were small, and the conclusions were contentious $(12,20)$. In our study, with 10-year of experience in the surgical treatment of AFMR, mid-term outcomes confirmed the efficacy of our technique. The overall 1-year and 3-year freedom from recurrent MR rates were $94.3 \%$ and $65.3 \%$, respectively. Consistently, $96.1 \%$ of patients were classified as NYHA functional class I/II without obvious heart failure symptoms during the mean follow-up time of $26.1 \pm 27.6$ months. Furthermore, LA and LV size was significantly reduced, suggesting that our surgical treatment can stop and even reverse cardiac remodeling. In terms of safety, we had no operative deaths or major complications in this study.

$\mathrm{AF}$ is the primary etiology and initial driver of AFMR and deserves attention during surgical management. It was reported that restoration of sinus rhythm by catheter ablation could mitigate MR and reverse-remodel LA (1). In a real-world setting, moderate-to-severe AFMR is often 
Table 4 Interaction between SA and preoperative LAD

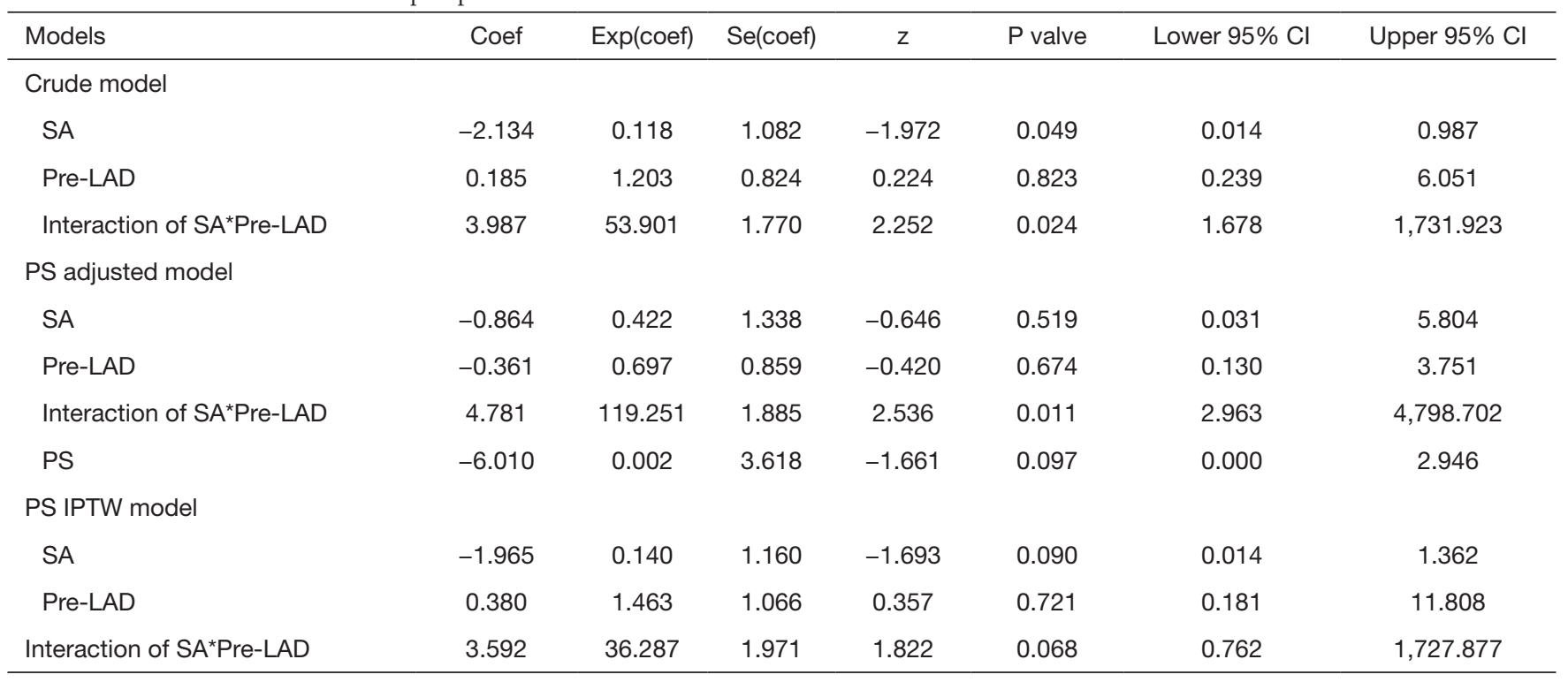

$\mathrm{Cl}$, confidence interval; IPTW, inverse probability of treatment weighting; LAD, left atrial diameter; Pre-LAD, preoperative LA diameter $>60 \mathrm{~mm}$ group; PS, propensity score; SA, surgical ablation group.

Table 5 Subgroup analysis

\begin{tabular}{lccccccc}
\hline Models & Coef & Exp(coef) & Se(coef) & z & P valve & Lower 95\% Cl & Upper 95\% Cl \\
\hline Pre-LAD $\leq 60$ mm subgroup: SA & -2.198 & 0.111 & 1.084 & -2.207 & 0.043 & 0.013 & 0.930 \\
Pre-LAD >60 mm subgroup: SA & 1.589 & 4.899 & 1.421 & 1.118 & 0.264 & 0.302 & 79.450 \\
\hline
\end{tabular}

$\mathrm{Cl}$, confidence interval; LAD, left atrial diameter; SA, surgical ablation group.

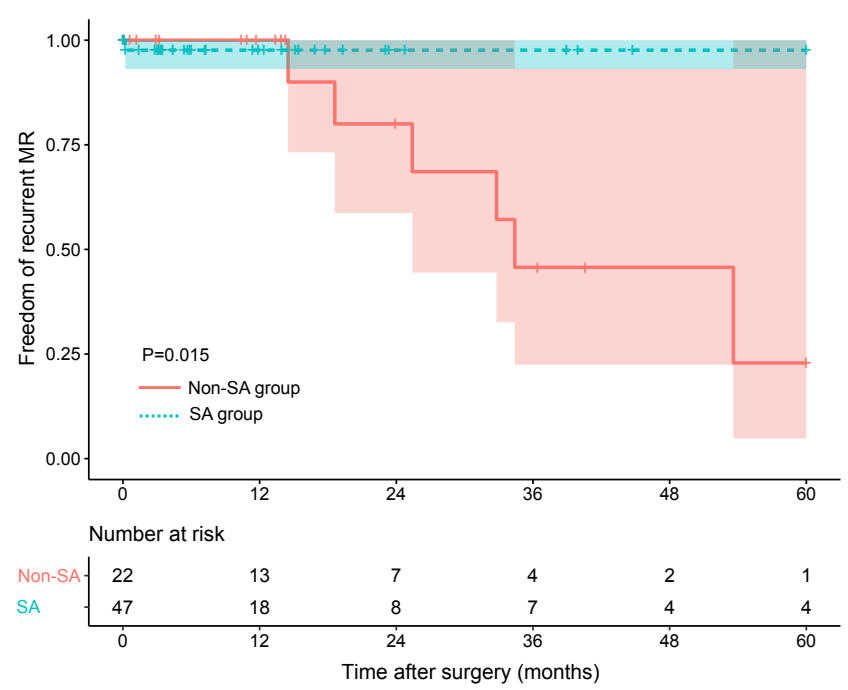

Figure 3 Kaplan-Meier curves depicting freedom from recurrent MR for the SA group and the non-SA group in the small LA group ( $\leq 60 \mathrm{~mm}$ ). Shadings indicate $95 \%$ confidence intervals. MR, mitral regurgitation; SA, surgical ablation; LA, left atrium. associated with long-standing persistent AF and a severely dilated LA and mitral annulus, meaning catheter ablation might be ineffective. Obviously, the decision making is influenced by potential surgical risks by adding Maze procedures and relatively low expectations of successful restoration of sinus rhythm in "sicker" patients. Vohra and colleague reported in their case series that only $7 / 20$ patients underwent a concomitant Maze procedure (12). In our study cohort, 52/82 patients received concomitant Maze procedures. A significant difference in recurrent $M R$ was found between the SA group and the non-SA group.

Furthermore, this significant effect of SA on recurrent MR was only found in the small LA group $(\leq 60 \mathrm{~mm})$ and not in the large LA group ( $>60 \mathrm{~mm}$ ) in the subgroup analysis. Appropriate patient selection is a pre-requisite for optimal surgical outcomes. Increased LA wall tension (21) and advanced LA fibrosis (22) may be associated with unsuccessful SA, while also increasing risk to patients. Our results suggest that adding Maze procedures to the standard surgical 
management of early stage AFMR can significantly help to restore sinus rhythm and achieve optimal long-term results.

For cardiologists or cardiac surgeons, a differential diagnosis with ventricular functional MR (VFMR) (ischemia MR or cardiomyopathy-related MR) is critical, due to the distinctive pathophysiological processes and surgical strategies of these two conditions. Leaflet tethering with the apical shift of the papillary muscle due to adverse LV remodeling and loss of normal leaflet coaptation is the principal pathological mechanism of VFMR. Chordalsparing MV replacement is now used, preferably over downsizing annuloplasty for VFMR according to the updated 2017 American Heart Association (AHA) guideline (23). AFMR patients usually have normal LV sizes, which is the key differentiator from VFMR. According to the results of the present study, MV repair with concomitant Maze procedure is safe and effective for AFMR.

This study had some limitations. Firstly, this was a single-center retrospective observational study with a relatively small number of patients, although this was the most extensive surgical study on AFMR in the literature. Secondly, the majority of patients underwent surgery in recent years, meaning that follow-up may be too short to assess the long-term outcomes of MV repair. Finally, the underlying mechanisms for the benefit of the concomitant SA procedure remain unclear.

\section{Conclusions}

We report on the safety and efficacy of MV repair for AFMR. Analysis of mid-term outcomes demonstrated improved heart failure symptoms and reverse-remodeling of both the LA and LV. A concomitant SA procedure may benefit patients in terms of recurrent MR, especially in the small LA group ( $\leq 60 \mathrm{~mm})$.

\section{Acknowledgments}

Funding: This work was sponsored by the Shanghai Sailing Program (No. 20YF1405400), the fellowship of China Postdoctoral Science Foundation (No. 2020M671001), and the National Natural Science Foundation of China (Grant Nos. 81801844, 81974272).

\section{Footnote}

Reporting Checklist: The authors present the study in accordance with the STROBE reporting checklist. Available at http://dx.doi.org/10.21037/atm-20-2958

Data Sharing Statement: Available at http://dx.doi. org/10.21037/atm-20-2958

Conflicts of Interest: All authors have completed the ICMJE uniform disclosure form (available at http://dx.doi. org/10.21037/atm-20-2958). The authors have no conflicts of interest to declare.

Etbical Statement: The authors are accountable for all aspects of the work in ensuring that questions related to the accuracy or integrity of any part of the work are appropriately investigated and resolved. The study was conducted in accordance with the Declaration of Helsinki (as revised in 2013). The study protocol was approved by the Ethics Committee of Zhongshan Hospital, Fudan University. Because of the retrospective nature of the research, the requirement for informed consent was waived.

Open Access Statement: This is an Open Access article distributed in accordance with the Creative Commons Attribution-NonCommercial-NoDerivs 4.0 International License (CC BY-NC-ND 4.0), which permits the noncommercial replication and distribution of the article with the strict proviso that no changes or edits are made and the original work is properly cited (including links to both the formal publication through the relevant DOI and the license). See: https://creativecommons.org/licenses/by-nc-nd/4.0/.

\section{References}

1. Gertz ZM, Raina A, Saghy L, et al. Evidence of atrial functional mitral regurgitation due to atrial fibrillation: reversal with arrhythmia control. J Am Coll Cardiol 2011;58:1474-81.

2. Delgado V, Bax JJ. Atrial Functional Mitral Regurgitation: From Mitral Annulus Dilatation to Insufficient Leaflet Remodeling. Circ Cardiovasc Imaging 2017;10:e006239.

3. Zoghbi WA, Adams D, Bonow RO, et al. Recommendations for Noninvasive Evaluation of Native Valvular Regurgitation: A Report from the American Society of Echocardiography Developed in Collaboration with the Society for Cardiovascular Magnetic Resonance. J Am Soc Echocardiogr 2017;30:303-71.

4. Kihara T, Gillinov AM, Takasaki K, et al. Mitral regurgitation associated with mitral annular dilation in patients with lone atrial fibrillation: an echocardiographic 
study. Echocardiography 2009;26:885-9.

5. Iwasaki YK, Nishida K, Kato T, et al. Atrial fibrillation pathophysiology: implications for management. Circulation 2011;124:2264-74.

6. Kagiyama N, Hayashida A, Toki M, et al. Insufficient Leaflet Remodeling in Patients With Atrial Fibrillation: Association With the Severity of Mitral Regurgitation. Circ Cardiovasc Imaging 2017;10:e005451.

7. Kilic A, Schwartzman DS, Subramaniam K, et al. Severe functional mitral regurgitation arising from isolated annular dilatation. Ann Thorac Surg 2010;90:1343-5.

8. Ito K, Abe Y, Takahashi Y, et al. Mechanism of atrial functional mitral regurgitation in patients with atrial fibrillation: A study using three-dimensional transesophageal echocardiography. J Cardiol 2017;70:584-90.

9. Takigawa M, Kuwahara T, Takahashi A, et al. The mechanism of mitral regurgitation assessed by preprocedural echocardiography is associated with the outcome of catheter ablation in patients with paroxysmal atrial fibrillation. J Interv Card Electrophysiol 2016;46:299-306.

10. Prabhu S, Voskoboinik A, Kaye DM, et al. Atrial Fibrillation and Heart Failure - Cause or Effect? Heart Lung Circ 2017;26:967-74.

11. Chatterjee NA, Chae CU, Kim E, et al. Modifiable Risk Factors for Incident Heart Failure in Atrial Fibrillation. JACC Heart Fail 2017;5:552-60.

12. Vohra HA, Whistance RN, Magan A, et al. Mitral valve repair for severe mitral regurgitation secondary to lone atrial fibrillation. Eur J Cardiothorac Surg 2012;42:634-7.

13. Calkins H, Kuck KH, Cappato R, et al. 2012 HRS/EHRA/ ECAS expert consensus statement on catheter and surgical ablation of atrial fibrillation: recommendations for patient selection, procedural techniques, patient management and follow-up, definitions, endpoints, and research trial design: a report of the Heart Rhythm Society (HRS) Task Force on Catheter and Surgical Ablation of Atrial Fibrillation. Developed in partnership with the European Heart Rhythm Association (EHRA), a registered branch of the European Society of Cardiology (ESC) and the European Cardiac Arrhythmia Society (ECAS); and in collaboration with the American College of Cardiology (ACC), American Heart Association (AHA), the Asia Pacific Heart Rhythm Society (APHRS), and the Society of Thoracic Surgeons (STS). Endorsed by the governing bodies of the American College of Cardiology Foundation, the American Heart Association, the European Cardiac Arrhythmia Society, the European Heart Rhythm Association, the Society of Thoracic Surgeons, the Asia Pacific Heart Rhythm Society, and the Heart Rhythm
Society. Heart Rhythm 2012;9:632-96.e21.

14. Badhwar V, Rankin JS, Damiano RJ Jr, et al. The Society of Thoracic Surgeons 2017 Clinical Practice Guidelines for the Surgical Treatment of Atrial Fibrillation. Ann Thorac Surg 2017;103:329-41.

15. Gu W, Guo H, Lu C, et al. Surgical ablation for persistent atrial fibrillation in concomitant cardiac surgery: mid-longterm result. Eur J Cardiothorac Surg 2017;52:888-94.

16. Damiano RJ Jr, Schwartz FH, Bailey MS, et al. The Cox maze IV procedure: predictors of late recurrence. J Thorac Cardiovasc Surg 2011;141:113-21.

17. Austin PC. The use of propensity score methods with survival or time-to-event outcomes: reporting measures of effect similar to those used in randomized experiments. Stat Med 2014;33:1242-58.

18. Kaneko H, Suzuki S, Uejima T, et al. Prevalence and the long-term prognosis of functional mitral regurgitation in Japanese patients with symptomatic heart failure. Heart Vessels 2014;29:801-7.

19. Dittrich HC, Pearce LA, Asinger RW, et al. Left atrial diameter in nonvalvular atrial fibrillation: An echocardiographic study. Stroke Prevention in Atrial Fibrillation Investigators. Am Heart J 1999;137:494-9.

20. Takahashi Y, Abe Y, Sasaki Y, et al. Mitral valve repair for atrial functional mitral regurgitation in patients with chronic atrial fibrillation. Interact Cardiovasc Thorac Surg 2015;21:163-8.

21. Wang W, Buehler D, Martland AM, et al. Left atrial wall tension directly affects the restoration of sinus rhythm after Maze procedure. Eur J Cardiothorac Surg 2011;40:77-82.

22. Kainuma S, Masai T, Yoshitatsu M, et al. Advanced left-atrial fibrosis is associated with unsuccessful maze operation for valvular atrial fibrillation. Eur J Cardiothorac Surg 2011;40:61-9.

23. Nishimura RA, Otto CM, Bonow RO, et al. 2017 AHA/ ACC Focused Update of the 2014 AHA/ACC Guideline for the Management of Patients With Valvular Heart Disease: A Report of the American College of Cardiology/ American Heart Association Task Force on Clinical Practice Guidelines. J Am Coll Cardiol 2017;70:252-89.

(English Language Editors: C. Betlazar-Maseh and J. Chapnick)

Cite this article as: Chen J, Wang Y, Lv M, Yang Z, Zhu S, Wei L, Hong T, Ding W, Lin Y, Wang C. Mitral valve repair and surgical ablation for atrial functional mitral regurgitation. Ann Transl Med 2020;8(21):1420. doi: 10.21037/atm-20-2958 